\title{
DESENVOLVIMENTO DE UMA FERRAMENTA PARA MAPEAR O PADRÃO DE AQUECIMENTO EM MICRO-ONDAS
}

\author{
J. A. SEBBEN ${ }^{1}$, L. M. RAYMUNDO ${ }^{1}$, F. C. BORGES ${ }^{1}$, C. M. SCHWEIBERGER ${ }^{1}$, J. da S. \\ ESPÍNDOLA $^{1}$ e J. O. TRIERWEILER ${ }^{1}$ \\ ${ }^{1}$ Grupo de Intensificação, Modelagem, Simulação, Controle e Otimização de Processos (GIMSCOP) \\ Departamento de Engenharia Química, Universidade Federal do Rio Grande do Sul \\ E-mail para contato: \{jasebben, raymundo, feborges, crisms, juliana, jorge \}@ enq.ufrgs.br
}

\begin{abstract}
RESUMO - O uso de aquecimento por micro-ondas é uma das peças chave da química verde. A energia das micro-ondas é transferida diretamente de forma rápida e simultânea para materiais capazes de absorvê-las. Contudo, a distribuição não-homogênea das microondas, a dificuldade na medição e o controle de temperatura, limitam o aproveitamento dessa tecnologia, sendo a homogeneidade do aquecimento um dos maiores desafios na sua aplicação. Devido à dificuldade de medição de temperatura em pontos diferentes da cavidade ressonante, desenvolveu-se uma sistemática para o mapeamento de aquecimento, a qual permite que se determine o perfil bidimensional em diferentes cotas da distribuição de energia por micro-ondas. O método consiste em utilizar grãos de pipoca localizados em diferentes pontos, os quais permitem que se acompanhe o padrão de aquecimento. Para diminuir a variabilidade da medição os grãos são pré-processados. Os resultados obtidos permitem encontrar a região de maior eficiência de aquecimento.
\end{abstract}

\section{INTRODUÇÃO}

A tecnologia das micro-ondas tem sido amplamente utilizada em processos de aquecimento, secagem, esterilização e pasteurização. Além disso, o aquecimento por micro-ondas tornou-se popularmente conhecido no processamento de alimentos devido à capacidade de aumentar a taxa de aquecimento com significativa redução no tempo de cozimento, manuseio seguro, fácil operação e baixa necessidade de manutenção (Chandrasekaran et al., 2011). Contudo, nos últimos anos, a aplicação das micro-ondas vem sendo explorada em diferentes áreas, transformando-a num dos verdadeiros trunfos da química verde, revolucionando setores como a síntese orgânica e a conversão de resíduos em produtos de melhor valor agregado.

Devido à enorme contribuição do aquecimento por micro-ondas, também chamado de aquecimento dielétrico, nos processos industriais, instigou-se aprofundar os conhecimentos já existentes referentes a esta tecnologia. Sendo a não-homogeneidade da distribuição de energia no interior de uma cavidade ressonante de um forno de micro-ondas, uma das motivações para a realização deste estudo. Assim, o objetivo do mesmo é mapear o padrão de aquecimento na cavidade. Visto que, esse estudo servirá como base para a adaptação de um forno comercial para processamento térmico de outros materiais, onde será necessário adaptar um reator, e por isso a análise do ponto de melhor aproveitamento energético. 


\subsection{Fundamentos do aquecimento dielétrico em um forno de micro-ondas}

Definem-se micro-ondas como as ondas eletromagnéticas com frequências entre 0,3 e $300 \mathrm{GHz}$ e comprimentos de onda entre 0,001 e $1 \mathrm{~m}$. São, portanto, ondas que estão entre a região das ondas de rádio e a região do infravermelho no espectro das ondas eletromagnéticas. A maioria dos reatores de micro-ondas para síntese química e fornos de micro-ondas domésticos opera a uma frequência de 2,45 $\mathrm{GHz}$, o que corresponde a um comprimento de onda de 12,25 cm (Schimink et al., 2011).

A formação das micro-ondas ocorre devido à interação de dois componentes perpendiculares, o campo elétrico e o campo magnético. Sendo que o aquecimento de determinadas substâncias é baseado na capacidade que as mesmas têm em absorver a energia das micro-ondas e efetivamente converter a energia eletromagnética em calor. (Motasemi et al., 2013). De acordo com a interação da radiação das micro-ondas com o material, existem três maneiras nas quais esses materiais podem ser caracterizados: (1) transparentes, que são os materiais pelos quais as micro-ondas atravessam sem que haja perdas, como teflon, quartzo e cerâmicas; (2) absorvedores, os que permitem a absorção das micro-ondas, como água e óleo, e (3) condutores, onde as micro-ondas não conseguem penetrar e por isso, são refletidas, a exemplo dos metais (Motasemi et al., 2013).

Conforme Mai et al. (2008) ondas confinadas entre paredes, como as micro-ondas na câmara de cozimento, sofrem reflexões que fazem com que haja ondas se deslocando em sentidos opostos. Estas ondas, de mesma frequência, mesma amplitude, mesma velocidade, mesma direção e sentidos opostos se sobrepõem. A superposição pode formar um padrão de ondas estacionárias, dependendo da relação entre o comprimento de onda e da distância entre as extremidades.

Sabe-se que onda transporta energia, mas no caso das ondas estacionárias isso não ocorre, pois os nodos não vibram, portanto, não transportam energia. Nesse caso a energia fica confinada nas regiões de vibração ocorrendo, periodicamente, conversões de energia cinética em energia potencial elástica e vice-versa. Por isso, num forno de micro-ondas, a câmara de cozimento é dimensionada de maneira que as suas paredes sempre coincidam com os nós das micro-ondas. Assim, praticamente não haverá absorção das ondas pelas paredes do forno, proporcionando reflexões próximas à condição ideal de formação de onda estacionária. Portanto, normalmente o alimento é colocado sobre um prato giratório para garantir uma distribuição uniforme de energia, pois se o alimento permanecesse estático, ter-se-iam pontos frios que coincidiriam com os nodos das ondas estacionárias (Mai et al., 2008), e hot spots nos pontos de maior intensidade do campo eletromagnético.

Com o intuito de comprovar a teoria das micro-ondas com a verdadeira distribuição de energia em um forno, Mehdizadeh (2010) estudou a capacidade que o método dos elementos finitos (FEM) tem em prever o comportamento do aquecimento pelas micro-ondas em uma cavidade ressonante. Sua metodologia consistiu na inserção de uma camada resistiva composta por material polimérico coberto por carbono, com geometria retangular e as mesmas dimensões internas do forno, o qual operou com a frequência de $2450 \mathrm{MHz}$ fornecida pelo magnetron. Em seu experimento, o autor utilizou uma folha de papel térmico de fax, a qual foi depositada acima da camada resistiva, sendo ambas envolvidas por duas finas camadas de vidro para assegurar um íntimo contato entre a camada resistiva e o papel térmico. Posteriormente, este aparato foi posicionado a uma altura de $5 \mathrm{~cm}$ da base inferior da cavidade interna do aparelho. Assim, com a mudança na coloração do papel, pode-se visualizar o padrão de aquecimento obtido. Os resultados alcançados por Mehdizadeh (2010) foram comparados com os obtidos pela modelo FEM simulado em COMSOL pelo mesmo autor, e, portanto, foi possível perceber uma grande concordância entre a localização dos hot spots - pontos de maior aquecimento entre os procedimentos realizados. 


\subsection{O desafio da termometria}

A medição de temperatura é um dos frequentes desafios encontrados no aquecimento eletromagnético. O mais comum e versátil sensor de temperatura é provavelmente o termopar. No entanto, o uso de termopares na presença de campo eletromagnético tem sido debatido, pois a natureza metálica dos elementos que compõem este sensor e seu revestimento, geralmente induz a perturbações na distribuição do campo eletromagnético. Consequentemente, estas distorções do campo podem provocar a formação de faíscas e interferir no sinal do termopar, fazendo com que os fios deste atuem como uma antena, transmitindo a interferência para outros instrumentos. (Leonelli et al., 2013; Mehdizadeh, 2010)

Assim, a utilização de termopares, ou elementos metálicos em geral, não é a melhor opção. Sendo preferível, se possível, o uso de fibras ópticas não perturbativas e métodos sem contato. O uso de métodos sem contato, como por exemplo, pirômetros ópticos, apresentam a desvantagem de que somente a temperatura da superfície do material é mensurada, além de não permitir a medição de um ponto, mas sim a média de uma superfície a qual aumenta quanto mais distante o sensor estiver do que está sendo medido, sendo assim, são ótimos para obter uma média de uma região em lugar de medidas pontuais. A principal vantagem dos pirômetros de infravermelho é permitir a medição não invasiva da temperatura, contornando os problemas oriundos dos outros métodos. Por exemplo, erros graves de medição surgem de efeitos causados pelo termopar, como a intensificação das micro-ondas em uma extremidade do sensor. Já as fibras ópticas também apresentam características negativas, tais como a delicadeza da fibra, a sensibilidade à degradação e à contaminação da sonda. Por esta razão, os pirômetros de infravermelho têm sido amplamente usados no aquecimento de materiais com microondas. (Leonelli et al., 2013; Luo et al.; 2013)

Se a temperatura no volume da carga deve ser conhecida, uma possível opção é o uso de fibras ópticas. Estes sensores são usualmente disponíveis para uma ampla faixa de temperatura, iniciando abaixo do ponto de congelamento da água, alcançando até $2000 \mathrm{~K}$. Porém, uma simples fibra óptica não é capaz de cobrir uma faixa inteira de temperatura. Logo, múltiplas fibras ópticas e controladores são requisitados. (Leonelli et al., 2013).

A desvantagem dos sensores de temperatura descritos é que nenhum fornece a distribuição de temperatura completa no volume total da amostra. Ou a temperatura é avaliada num ponto, ou na superfície. Dessa forma, percebe-se que a zona para medição de temperatura deve ser cuidadosamente escolhida, possivelmente com a ajuda de simulações numéricas ou outras ferramentas, a fim de prever quais regiões da cavidade ressonante de um forno de micro-ondas terá a maior incidência de radiação. Em vista disso, percebe-se que outro desafio da termometria no aquecimento eletromagnético é que a medição de temperatura em uma localização específica não garante necessariamente que a temperatura em outros locais seja equivalente, a menos que se tenha o cuidado de conhecer o perfil de temperatura previamente. (Leonelli et al., 2013; Mehdizadeh, 2010)

Conhecendo as limitações existentes para o melhor aproveitamento energético em vários pontos da cavidade interna de um forno de micro-ondas, buscou-se uma alternativa eficiente para a realização da mesma. Assim, encontrou-se nos grãos de milho pipoca uma possível solução para o problema aqui descrito, uma vez que os grãos podem ser tratados como integradores energéticos, não estáticos, possibilitando a realização de análises quantitativas do poder de absorção das micro-ondas pelas amostras.

O milho pipoca (Zea mays L.) é um tipo de milho que tem como principal característica, grãos pequenos e duros que apresentam a capacidade de estourar devido a uma pressão de 135 psi formada 
dentro do grão quando aquecidos em torno de $177{ }^{\circ} \mathrm{C}$ (Hoseney et al., 1983). Segundo Silva (1993) a expansão ocorre devido à resistência do pericarpo associado ao teor de umidade e teor de óleo do grão. Quando submetido ao calor, o amido presente no milho pipoca expande-se, e a água contida no interior do grão transforma-se em vapor d'água, aumentando gradualmente a pressão interna do grão, até que a casca não aguenta a pressão e ocorre a explosão.

\section{FERRAMANTA DE MAPEAMENTO DE AQUECIMENTO}

\subsection{Preparo das amostras}

Utilizou-se no experimento, milho para pipoca Tipo 1 da Yoki Alimentos Ltda. O teor de umidade médio dos grãos comercializados está entre 10 e $15 \%$.

Um pré-tratamento das amostras foi realizado a fim de padronizar o teor de umidade contido nas amostras utilizadas no experimento. Para esse fim, foram adicionados $200 \mathrm{~mL}$ de água à temperatura de $23{ }^{\circ} \mathrm{C}$ em um recipiente contendo 500 grãos de milho-pipoca. Foi estabelecido o tempo de 60 minutos de imersão dos grãos, sem agitação e aquecimento.

Para determinar o teor de umidade dos grãos pré-tratados, amostras compostas por 100 grãos foram acondicionadas em estufa à temperatura de $105^{\circ} \mathrm{C}$ por um período de $24 \mathrm{~h}$. Percebeu-se que este tempo foi suficiente, pois a partir de tal, não houve mais variação de massa nas amostras. Um teor

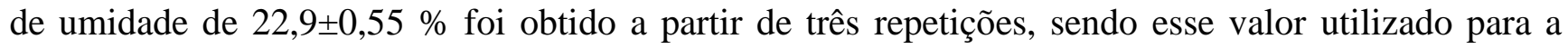
realização dos experimentos de mapeamento de radiação na cavidade ressonante.

\subsection{Desenvolvimento da ferramenta}

Para a realização dos experimentos de aquecimento dielétrico, foi utilizado um forno de microondas da marca LG classe Intellwave Grill modelo MB-274PL, com frequência operacional de 2450 $\mathrm{MHz}$ e potência de consumo elétrico de aquecimento por micro-ondas de $1050 \mathrm{~W}$. As dimensões internas do forno são $30 \mathrm{~cm}$ de largura; 19,5 de altura e 28,5 cm de profundidade.

A sistemática desenvolvida para mapear o padrão de aquecimento dielétrico no interior da cavidade ressonante de um forno de micro-ondas doméstico consistiu da divisão do interior da cavidade ressonante em 27 regiões distintas. Primeiramente, foram estabelecidos 3 planos na horizontal, diferenciados verticalmente pela altura em relação à base, como pode ser visualizado pelo desenho da Figura 1. O plano 1, refere-se à base inferior da cavidade, já o plano 2, à parte intermediária, distanciado $6 \mathrm{~cm}$ da base e por último, na parte superior, o plano 3, com uma distância de $13 \mathrm{~cm}$ da base da cavidade. Posteriormente cada um dos planos foi dividido em nove regiões denominadas de 11 à 39, referindo-se o primeiro algarismo ao nível do plano e o segundo às posições, conforme mostrado nos esquemas da Figura 2.

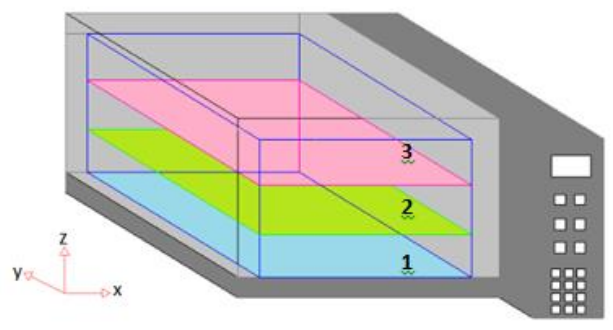


Cada uma das 27 regiões definidas continham igualmente $63 \mathrm{~cm}^{2}$, sendo que em cada uma delas foi adicionado um suporte plástico subdivido em quatro compartimentos de igual tamanho. Então, em cada um desses compartimentos foram alocados 25 grãos, e como são quatro compartimentos por região, totalizou-se uma quantidade de 100 amostras por região.

O aquecimento por micro-ondas foi acionado em potência máxima, permanecendo ligado por um período de 5 minutos. Ao término do aquecimento, contabilizou-se o número de grãos de milhopipoca não estourados. Os ensaios foram realizados para cada região individualmente e por se tratar de um experimento com diversos fatores que influenciam na confiabilidade dos resultados, os experimentos foram reproduzidos em triplicata.
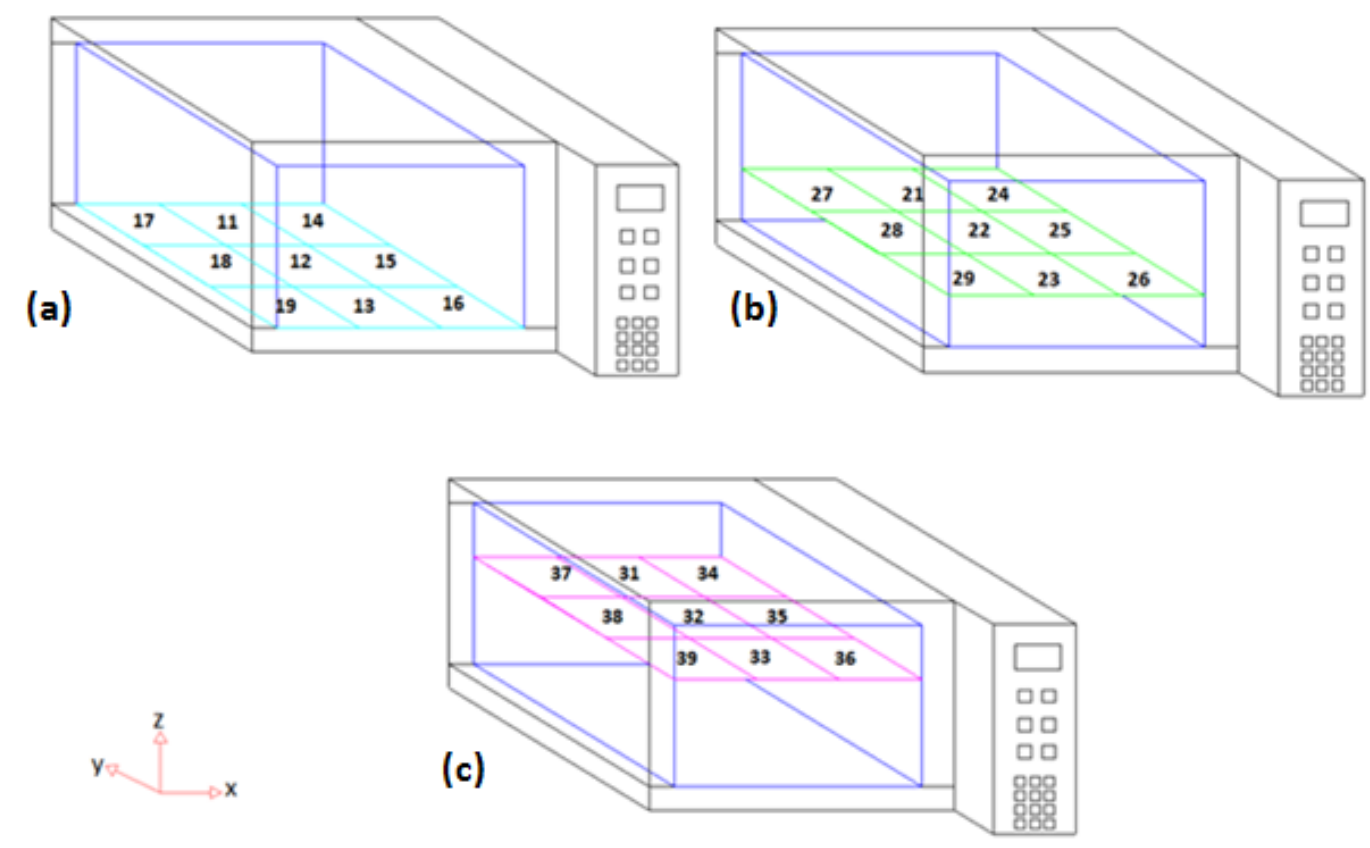

Figura 2 - Ilustração da cavidade interna de um forno de micro-ondas divida nas diferentes regiões experimentais. (a) Plano 1; (b) Plano 2 e (c) Plano 3.

Devido à natureza explosiva da amostra quando ocorre a ruptura de sua parede, o procedimento foi cuidadosamente acompanhado, a fim de que todos os grãos não estourados permanecesse na sua posição de origem.

Após a realização dos experimentos, realizou-se uma análise de variância (ANOVA), a qual é um teste estatístico que mostra se existe alguma diferença significativa entre diferentes grupos. Na ANOVA realizada neste trabalho, utilizou-se o método de Tukey, o qual é um teste de causa e efeito para determinar quais grupos são significativamente diferentes entre si. Os componentes de uma tabela ANOVA são os graus de liberdade de cada fonte (DF), a soma dos quadrados entre as regiões e os erros (SS), a média dos quadrados encontrada pela divisão da soma dos quadrados pelos graus de liberdade (MS), a divisão da variância entre as regiões pela variância entre os erros (F) e o valor para determinar a significância do fator $(\mathrm{P})$. 


\section{RESULTADOS E DISCUSSÕES}

Com o auxílio do software Minitab, realizou-se uma análise de variância para o fator posição, com o objetivo de testar a equabilidade das médias categorizadas por um único fator. Os resultados desta análise podem ser visualizados na Tabela 1.

Tabela 1 - Análise de variância

\begin{tabular}{llllll}
\hline Fonte & DF & SS & MS & F & P \\
\hline Região & 26 & 35033,6 & 1347,4 & 20,38 & 0,00000 \\
Erros & 54 & 3570,7 & 66,1 & & \\
Total & 80 & 38604,2 & & & \\
\hline
\end{tabular}

$\mathrm{O}$ valor $\mathrm{P}$ de 0,00000 indica que o fator região é significativo e que as diferenças entre os resultados são reais e dependem unicamente dos mesmos.

A Tabela 2 fornece a informação de agrupamento usando o método de Tukey e 95,0\% de confiança. Também são fornecidos os valores médios das amostras que estouraram nas respectivas posições.

O agrupamento obtido significa que as regiões que apresentam a mesma letra são semelhantes entre si. Por exemplo, todas as regiões que possuem a letra A,B,C,D,E,F,G,H ou I no seu agrupamento são similares e ainda, no caso das regiões $28,12,29$ e 25, o mesmo agrupamento $\mathrm{ABC}$ gerado pela análise, permite afirmar que não há diferenças significativas entre as mesmas.

Tabela 2. Quantidade média de amostras transformadas e agrupamento das regiões

\begin{tabular}{lllllllr}
\hline Região & Média & $\begin{array}{l}\text { Desvio } \\
\text { Padrão }\end{array}$ & Agrupamento & Região & Média & $\begin{array}{c}\text { Desvio } \\
\text { Padrão }\end{array}$ & Agrupamento \\
\hline $\mathbf{3 8}$ & 90,000 & 7,550 & A & $\mathbf{1 9}$ & 41,667 & 9,866 & C D E F G \\
$\mathbf{1 1}$ & 72,000 & 4,359 & A B & $\mathbf{3 5}$ & 36,333 & 1,155 & D E F G \\
$\mathbf{2 8}$ & 66,333 & 5,033 & A B C & $\mathbf{1 4}$ & 35,333 & 8,021 & E F G H \\
$\mathbf{1 2}$ & 65,667 & 17,098 & A B C & $\mathbf{1 5}$ & 28,333 & 2,082 & F G H I \\
$\mathbf{2 9}$ & 64,667 & 7,506 & A B C & $\mathbf{2 2}$ & 24,333 & 2,517 & G H I \\
$\mathbf{2 5}$ & 64,667 & 6,658 & A B C & $\mathbf{3 9}$ & 24,000 & 7,000 & G H I \\
$\mathbf{1 8}$ & 62,000 & 19,079 & B C D & $\mathbf{1 6}$ & 22,667 & 0,577 & G H I \\
$\mathbf{2 7}$ & 58,333 & 1,528 & B C D E & $\mathbf{3 6}$ & 21,000 & 3,464 & G H I \\
$\mathbf{3 1}$ & 56,000 & 13,115 & B C D E & $\mathbf{2 1}$ & 21,000 & 5,292 & G H I \\
$\mathbf{3 7}$ & 55,667 & 7,506 & B C D E & $\mathbf{2 4}$ & 20,000 & 7,211 & G H I \\
$\mathbf{1 3}$ & 54,333 & 11,719 & B C D E & $\mathbf{2 3}$ & 18,667 & 5,686 & G H I \\
$\mathbf{2 6}$ & 48,000 & 2,646 & B C D E F & $\mathbf{3 3}$ & 9,333 & 0,577 & H I \\
$\mathbf{3 4}$ & 42,667 & 9,074 & C D E F G & $\mathbf{3 2}$ & 8,333 & 3,055 & I \\
$\mathbf{1 7}$ & 42,333 & 11,846 & C D E F G & & & & \\
\hline
\end{tabular}

Conforme o agrupamento obtido, pode-se perceber que as regiões que compõe o grupo A (38, $11,28,12$ e 29) correspondem às zonas onde houve a absorção da energia necessária para o rompimento dos grãos para uma maior quantidade de amostras, portando, supõe-se que sejam as 
regiões de maior incidência de micro-ondas no interior da cavidade ressonante. $\mathrm{O}$ contrário pode ser dito para o grupo I, composto pelas regiões $15,22,39,16,36,21,24,23,33$ e 32.

As comparações e a quantificação do número de grãos estourados podem ser vistos na Figura 3. Nesta figura, a posição das colunas condiz com a posição das regiões conforme apresentado na Figura 2. Logo, entende-se que as posições da porta e da parede direita do forno, estejam representadas pelas letras $\mathrm{x}$ e y respectivamente.

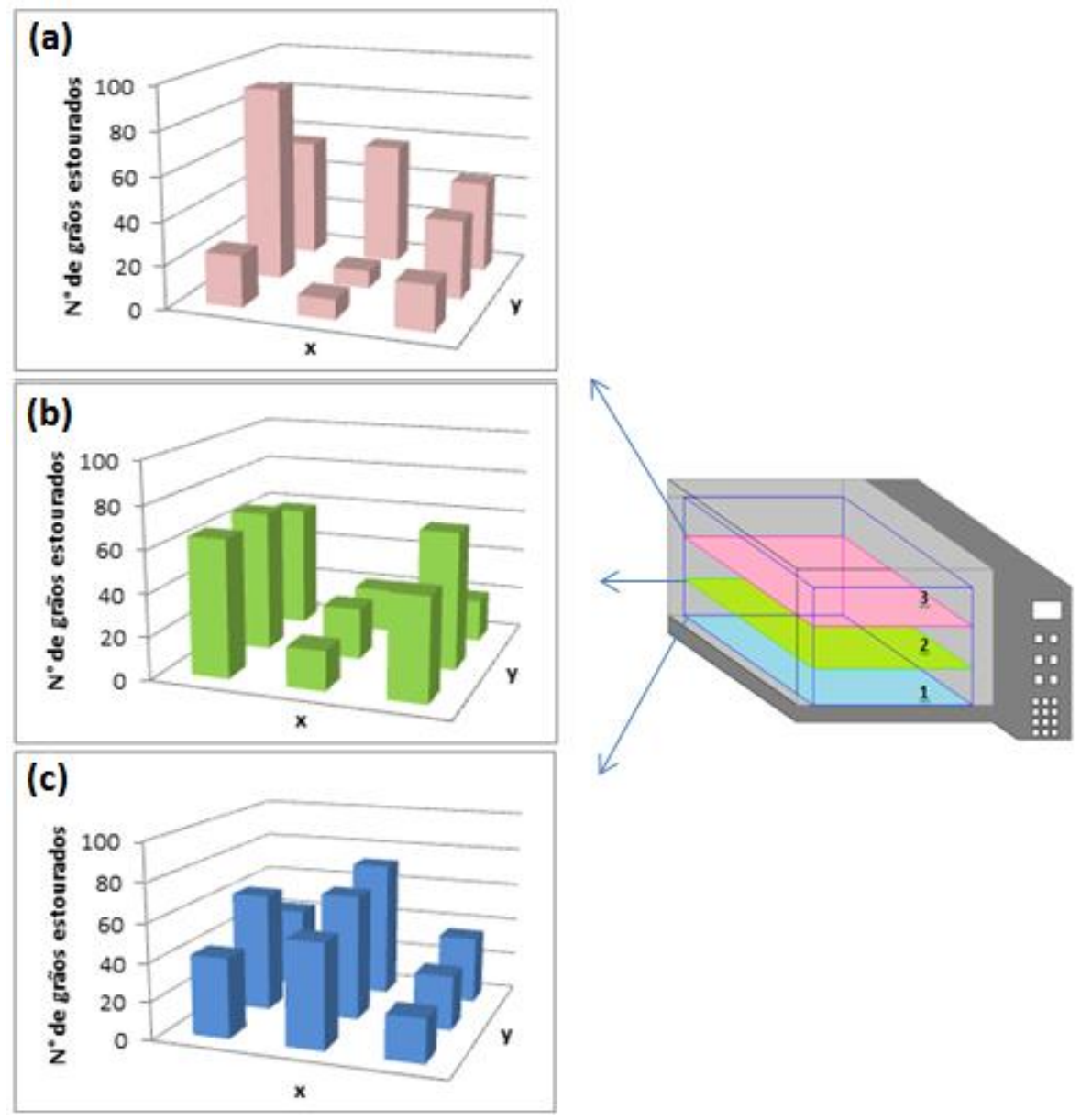

Figura 3 - Quantidade de amostras que absorveram energia suficiente para estourar nas 27 regiões da cavidade ressonante. Entende-se ' $x$ ' como a posição frontal, onde fica a porta, e ' $y$ ' da parede direita do forno. (a) Plano 3 - superior; (b) Plano 2 - intermediário e (c) Plano 3 -inferior.

Com os resultados apresentados, percebe-se a não-homogeneidade do aquecimento na cavidade ressonante. Consequentemente, a amplitude desses valores influenciará no posicionamento e geometria de um reator que será adaptado no interior de um forno de micro-ondas comercial, que servirá como base para outros estudos realizados pelo grupo de pesquisa envolvido neste trabalho.

Os resultados obtidos nesse estudo como a intensidade da energia das micro-ondas em diferentes posições da cavidade interna de um forno retangular, também podem ser comparados à distribuição de energia demonstrada por Medizadeh (2010). Em seu trabalho, o autor conclui que a uniformidade da densidade de energia é muito baixa, pois em poucos centímetros esta densidade passa de um valor máximo a um valor mínimo. No entanto, apesar da semelhança entre as conclusões obtidas no trabalho publicado por Medizadeh( 2010 ) verificou-se que a estratégia de adotar grãos de milho pipoca como
Area temática: Simulaçao, Otimizaçao e Controle de Processos 
integradores energéticos é um diferencial e apresenta vantagens às demais, devido à natureza não estática dos grãos e a possibilidade da quantificação dos resultados de maneira rápida, fácil e dinâmica.

\section{CONCLUSÕES}

Com a metodologia desenvolvida se pode atestar que a distribuição da energia das micro-ondas na cavidade interna de um forno de micro-ondas doméstico é realmente irregular. Sendo que a utilização de grãos de milho de pipoca, cuja peculiar transformação na sua estrutura física ocorre devido ao processo de absorção de energia, mostrou-se uma alternativa rápida, visual e coerente para relacionar as diferentes posições na cavidade ressonante com o potencial de aquecimento local. A principal vantagem da metodologia desenvolvida é que ela permite que se estude o efeito de partículas em movimento no processo aquecimento com micro-ondas. Essa característica é fundamental quando se quer estudar o processo de aquecimento em sistemas não estáticos, como no caso de leitos fluidizados. Nessas situações técnicas clássicas não seriam capazes de determinar esse efeito de aquecimento. Outra vantagem da presente proposta consiste na dimensão das partículas, as quais também são adequadas para o estudo de partículas em movimento.

\section{REFERÊNCIAS}

CHANDRASEKARAN, S.; RAMANATHAN, S.; BASAK, T. Microwave food processing - A review. Food Research International, 2013.

HOSENEY, R.C.; ZELEZNAK,K.; ABDELRAHMAN, A. Mechanism of Popcorn popping. Journal of Cereal Science I, p. 43-52, 1983.

LEONELLI, C.; VERONESI, P.; CRAVOTTO, G. Microwave-Assisted Extraction: An Introduction to Dielectric Heating. In: (Ed.). Microwave-assisted Extraction for Bioactive Compounds: Springer, 2013. p.1-14. ISBN 1461448298.

LUO, S.D.; YANG, Y.F.; SCHAFFER, G.B.; QIAN, M.; Calibration of Temperature Measurement by Infrared Pyrometry in Microwave Heating of Powder Materials: an Exothermic Reaction Based Approach. Journal of Microwave Power and Electromagnetic Energy, 47 (1), p. 5-11, 2013.

MAI, I.; BALZARETTI, N. M.; SCHMIDT, J. E. Utilizando um forno de micro-ondas e um disco rígido de um computador como laboratório de física. v.18, n.6, Porto Alegre, 2008.

MEHDIZADEH. M. Microwave/RF Applicators and Probes for material heating, sensing and plasma generation: Elsevier, 2010.

MOTASEMI, F.; AFZAL, M. T. A review on the microwave-assisted pyrolysis technique. Renewable and Sustainable Energy Reviews, v.28, p. 317-330, 2013.

SCHIMINK, J.R.; LEADBEATER, N.E. Microwave Heating as a Tool for Sustainable Chemistry: An Introduction. Microwave Heating as a Tool for Sustainable Chemistry: CRC Press, 2011.

SILVA, W.J. Estudo amplia pesquisa de milho. Jornal da UNICAMP, p. 8, 1993. 